\title{
Segmental nature of the choroidal vasculature*
}

\author{
SOHAN SINGH HAYREH \\ From the Department of Ophthalmology, University of Iowa, Iowa City, Iowa, USA
}

The classical textbook description of the choroidal vasculature was well summed up by Hogan, Alvarado, and Weddell (197r) when they wrote that, in the choroid, 'extensive anastomoses exist between the various branches of all the short ciliary arteries, so that occlusion of one vessel ordinarily does not produce infarction of the choroid'. It is also well known that inflammatory, metastatic, and degenerative lesions in the choroid are usually localized. Duke-Elder (196I) commented: 'The tendency for inflammatory and degenerative diseases of the choroid to show a considerable degree of selective localization, despite the fact that anatomically the vessels would appear to form a continuous network, has given rise to speculations regarding the anatomical isolation of specific choroidal areas'.

Over the past few years I have investigated the choroidal vascular bed anatomically and clinically (in the human) and experimentally (in rhesus monkeys). These studies have been reported from time to time piecemeal (Hayreh, 1962-1975), but in this paper an attempt is made to summarize concisely the overall results of my investigations. My various studies can be likened to pieces of a jig-saw puzzle; now by fitting all the pieces together, we can, I believe, arrive at a much clearer insight into the true pattern of the choroidal blood supply. My observations show that the posterior ciliary arteries and their branches, as well as the vortex veins, have a segmental distribution in the choroid, and that the choroidal arteries are end-arteries.

\section{Arterial supply of the choroid}

ANATOMY OF THE POSTERIOR CILIARY ARTERIES

The earlier textbook descriptions of the posterior ciliary arteries (PCAs) tend to be misleading and there has been much confusion about their intraorbital course. For example, Duke-Elder (196r) stated: 'These arteries have a variable origin from

*Part of this work was supported by a research grant from the Medical Research Council, London

Requests for reprints: S. S. Hayreh, Department of Ophthalmology, University of Iowa Hospitals, Iowa City, Iowa 52242, USA the ophthalmic artery within the orbit, and may be subdivided into two groups-the short and the long'.

A detailed account of my studies of the anatomy of the PCAs in man is given elsewhere (Hayreh, 1962); the ophthalmic artery gives out not short and long PCAs, but parent trunks (mostly two or three in number). These I have designated the 'PCAs', either medial (MPCA) or lateral (LPCA) depending upon their relationship with the optic nerve at the point of penetration of their branches into the sclera (Fig. I). Occasionally an additional superior PCA may be seen (Meyer, 1887; Hayreh,

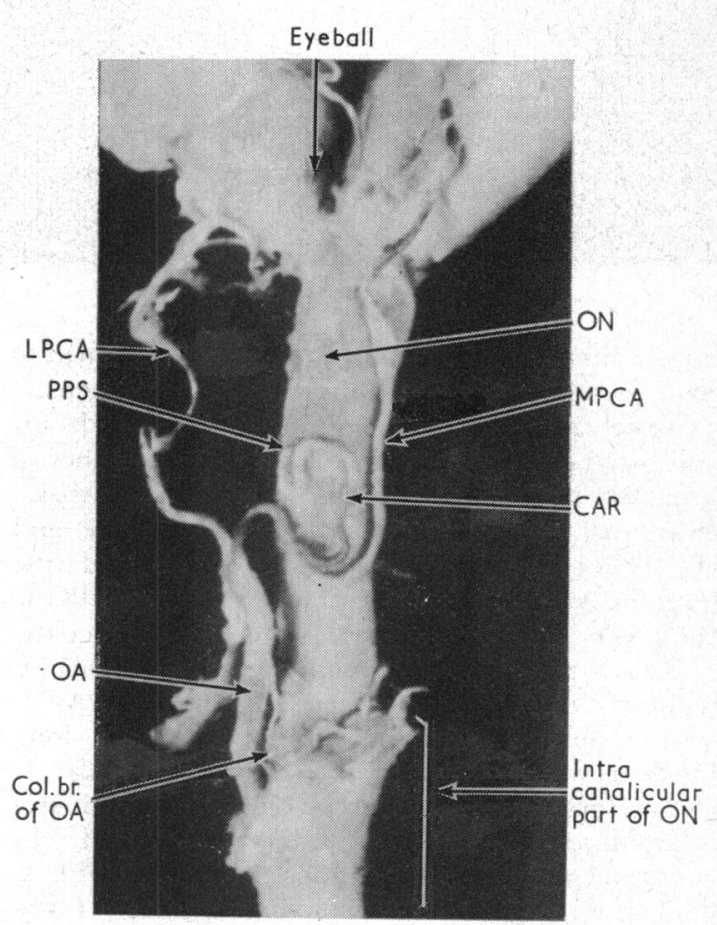

FIG. I Photograph of inferior surface of optic nerve $(O N)$ and eye ball, showing ophthalmic artery (OA), central artery of retina (CAR), medial (MPCA) and lateral (LPCA) posterior ciliary arteries filled with Neoprene latex. Also shown are collateral branches (Col. br.) of $O A$ to intracanalicular part of $O N$ 
1962), although Weiter and Ernest (1974) claimed to have seen it in over one-third of their specimens.

From their origin, the PCAs run forwards towards the eyeball and divide into a large number of branches before piercing the sclera. Out of these, two small branches (one on the medial side and the other on the lateral side) are called the long PCAs, while the rest are the short PCAs (SPCAs). The temporal SPCAs generally pierce the sclera in the area corresponding to the macular region. The intrascleral course of the SPCAs is straight and very short, while that of the long PCAs is oblique and long (Hayreh, r974d). It is therefore important that we clearly define in any description the type of artery concerned. In almost all previous descriptions ' $\mathrm{PCA}$ ' and 'SPCA' have been considered synonymous, and yet the former is the mother and the latter one of the many daughters (the long PCA being another daughter)!

My experimental studies were carried out in rhesus monkeys; the normal anatomical pattern of the PCAs in the monkeys (Hayreh, 1964) revealed that there is usually one large LPCA and sometimes two, usually two MPCAs and sometimes one or three. This pattern was similar to that seen in man.

DISTRIBUTION IN THE CHOROID BY THE VARIOUS ARTERIES

Posterior ciliary arteries (PCA)

The MPCA and LPCA usually supply the nasa and temporal halves respectively of the choroid (Figs 2, 3). LPCA may supply up to two-thirds of the choroid with MPCA supplying a correspondingly smaller area (Hayreh, r970; Hayreh and Baines, I972a) (Fig. 4). In some instances the area of supply by the MPCA and LPCA may be somewhat obliquely oriented (Fig. 3), In 20 per cent of the eyes in rhesus monkeys I found that, instead of the lateral and medial distribution, there was a superior and inferior distribution (Hayreh, 1970) (Fig. 5). The border between the area of supply by the MPCA and LPCA usually passes through the optic disc (Figs 2 to 5). When the number of either LPCAs or MPCAs is more than one, the choroid supplied by the PCA may be a 


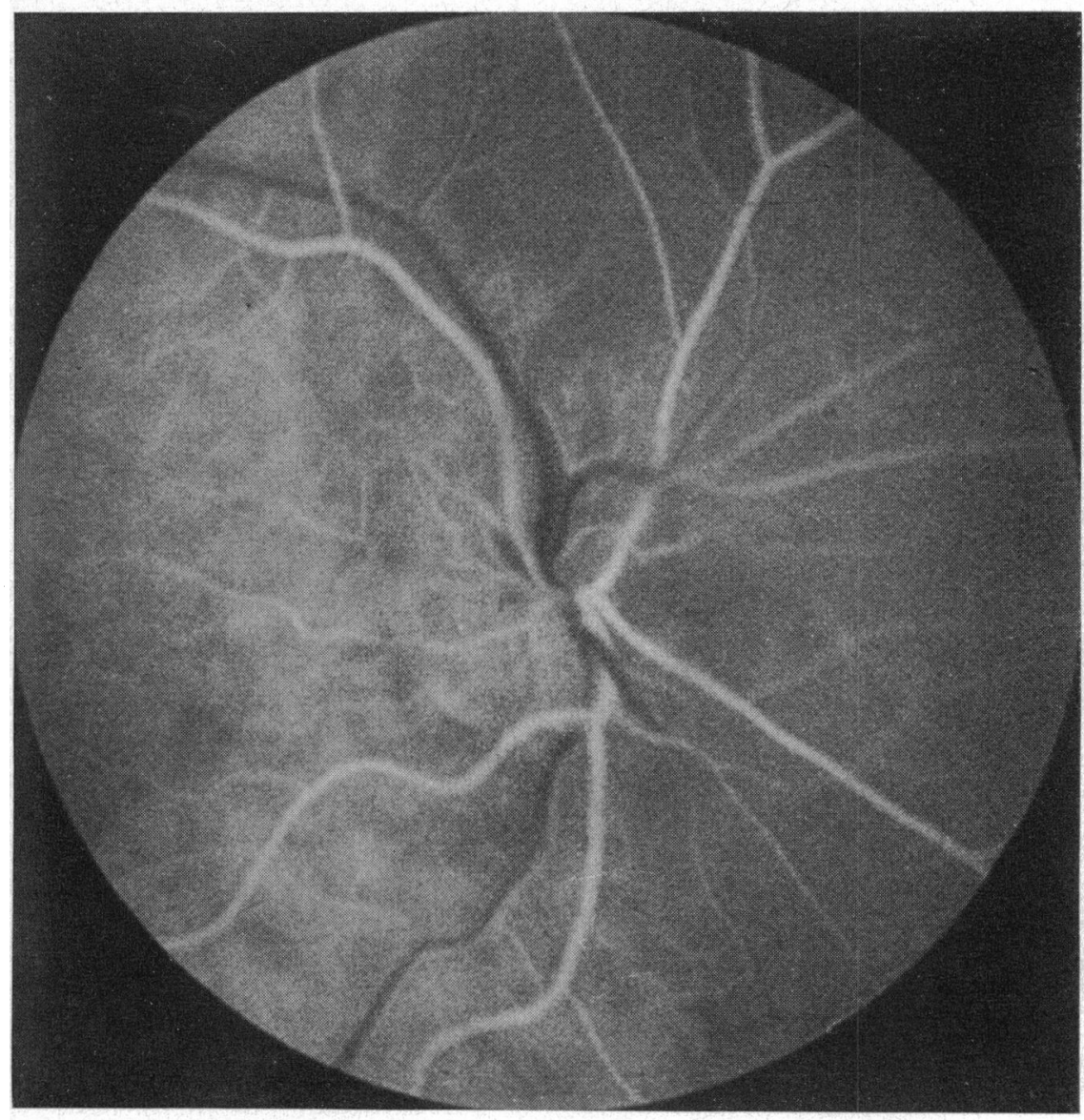

FIG. 3 Fluorescein fundus angiogram during retinal arterial phase of right eye of 76-year-old woman, with anterior ischaemic optic neuropathy of superiornasal half of optic disc and temporal arteritis, showing non-filling of superiornasal half of choroid and optic disc

segment of the usual distribution by the medial or lateral PCA (Fig. 6).

\section{Short posterior ciliary arteries (SPCA)}

These were investigated only in the area of the LPCA (Hayreh, 1974c). The area of the choroid supplied by each temporal SPCA varies greatly in size, shape, and location; and these segments resemble a jig-saw puzzle in the distribution of the LPCA (Fig. 7). Each piece of the jig-saw pattern has a well-defined margin which forms the watershed zone between the adjacent SPCAs. Since the temporal SPCAs pierce the sclera to enter the eyeball in the macular region and spread out to the periphery of the fundus radially to supply the temporal half of the choroid it is natural that most of the segments of the choroid supplied by the temporal SPCAs and their watershed zones should meet in the macular region and extend from the posterior pole to the periphery (Fig. 7). Smaller sub-divisions of the SPCAs supply still smaller segments of irregular shape and size with well-defined margins.
These are frequently seen on fluorescein angiography as geographical spatial filling defects in the normal human choroid (Fig. 8). Each of the terminal choroidal arterioles supplies a small segment of choriocapillaris, and these are arranged like a mosaic (Fig. 9).

\section{Long posterior ciliary artery (long PCA)}

My studies revealed that one long PCA branches from the MPCA and the other from the LPCA: they do not arise directly from the ophthalmic artery; this contradicts the classical textbook description (Leber, I 903; Wolff, 1948; Duke-Elder, r 96 I).

Once again, contrary to the accepted description of distribution in the choroid by the long PCA, my studies (Hayreh, 1974d) have revealed that the temporal long PCA invariably supplies a sector of the choroid posterior to the equator, starting almost immediately from the point where it joins the choroid after it has pierced the sclera (Fig. 7) and extending forwards. Weiter and Ernest (1974) 
also described a similar distribution in human eyes.

\section{Is there a special macular artery supplying the submacular choroid?}

To explain the selective localization of many pathological lesions in the macular region, the existence of such an artery has been postulated by Hepburn (1912) and Amalric (1973) but not on an anatomical basis. Weiter and Ernest (1974), from studies involving intravascular injection of both Indian ink and Neoprene latex into 67 human cadaver eyes, described the invariable presence of a discrete submacular choroidal circulation provided by a direct submacular artery and a recurrent submacular branch of the temporal long PCA. Using identical injection methods in human eyes, Wybar (1954a) (27 eyes) and Ring and Fujino ( 1967 ) (125 eyes) failed to find any such artery in their meticulous studies. It is worth noting that Wybar (1954a, b) was specifically interested in exploring the submacular blood supply and yet failed to find what Weiter and Ernest (1974) claimed
FIG. 4 Fluorescein fundus angiogram of right eye of monkey after experimental occlusion of central retinal artery. Lateral posterior ciliary artery supplies temporal half and superior nasal part of choroid. Early filling of medial posterior ciliary artery in inferior nasal sector of choroid is seen. Border zone between these and nasal peripapillary region is still not filled. Part of optic disc supplied by lateral posterior ciliary artery is filled to have seen in all of their 67 eyes. It is difficult to understand how all the previous workers investigating the submacular choroid by the same technique could have failed to find what Weiter and Ernest (1974) claimed to be a constant feature. In my experimental study in 47 eyes of rhesus monkeys, in which my primary objective was to investigate the submacular choroidal blood supply, I did not find any submacular artery (Hayreh, 1974c). In spite of the claims of Weiter and Ernest (I974) to have discovered a special submacular artery (although little definite evidence is offered in their paper), there is so far a preponderance of evidence against the existence of such an artery. The subject of the submacular choroidal vasculature is discussed at length elsewhere (Hayreh, 1974c).

\section{Choriocapillaris}

The textbook description of the choriocapillaris is that it forms a single continuous layer of wide-lumen capillaries arranged in one plane, and that these form a continuous anastomotic network over the entire choroid. Although Rohen (1965) stated that 


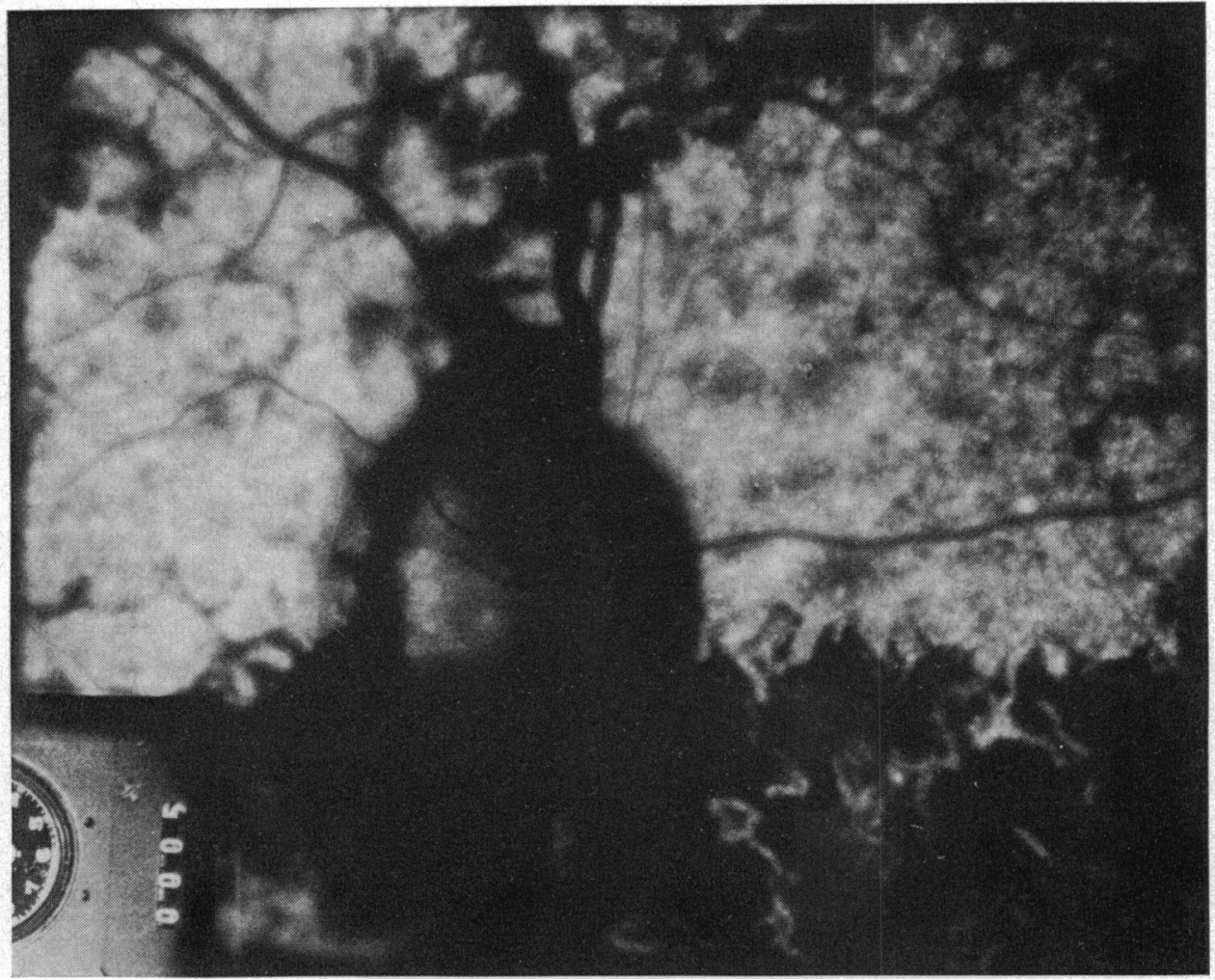

FIG. 5

Fluorescein fundus angiograms of right eye of monkey after experimental occlusion of central retinal artery, showing filling of upper half of choroid and optic disc with well-demarcated horizontal border 'the entire choriocapillaris network undoubtedly has a continuity by capillary anastomoses', he did find that 'the finer branches of the choroidal arteries run in relatively delimited sectors of the choriocapillaris'. Henkind (I 967) stated that 'blocking a choroidal artery leads to diminished or absent flow in a large segment of the choriocapillaris; this is in spite of the fact that the choriocapillaris seems to be a continuous anastomostic network over the entire choroid'. Dollery, Henkind, Kohner, and Paterson (1968) described fluorescein angiography of pigs' eyes with experimentally raised intraocular pressure; they recorded the filling of the choriocapillaris in the form of dots, and stated that 'this would indicate that the choriocapillaris fills as a series of small independent segments rather than as a continuum over the entire surface'.

My fluorescein angiographic studies on the choriocapillaris (Hayreh, 1974b) revealed that each terminal choroidal arteriole supplied an independent segment of the choriocapillaris, with the arteriole joining the segment in its centre, and the drainage venules lying around the periphery of this segment (Fig. IO). Each segment is a functional unit, usually of a polygonal shape (Fig. 9) with no anastomosis with the adjacent segments in vivo. The various segments are arranged like a mosaic, the borders of the mosaic being formed by the venous channels (Figs 9, 10). This has been confirmed by the finding of a lobular arrangement in the choriocapillaris, in a study of flat preparations of the choriocapillaris (Torczynski and Tso, 1974). The size of each unit of the mosaic varies, and it was found that the diameter of each unit was usually about one-quarter of the disc diameter or less (Figs 2, 9). Krey (1975) recently confirmed the segmental distribution by the choriocapillaris in man, by using the histochemical alkaline phosphatase reaction in cadaver eyes. He stated, however, that his technique showed that the feeding arteriole was at the periphery and the draining venule in the centre of the choriocapillaris lobule. This picture of the feeding arteriole and draining venule in a choriocapillaris lobule is the reverse of that seen not only by me on fluorescein angiography in the living eye but also on fluorescein angiography by Dollery, Henkind, Kohner, and Paterson (1968) and others, on flat preparation examination of the choriocapillaris by Torczynski and Tso (1974), and on injection studies in cadaver eyes by Weiter and Ernest (1974), and many others. The filling pattern sequence of the vascular bed on fluorescein angiographic studies (Fig. 9) shows the state in the living eye while histochemical staining techniques do not give the same information and can be misleading. Unfortunately, we 
already know that post-mortem studies in the choroidal vascular bed are markedly inferior to in vivo studies to obtain information about the state of the circulation in the living eye and have given rise to some misleading artefacts.

\section{CHOROIDAL ARTERIAL ANASTOMOSES}

The classical concept was well summed up by Ring and Fujino (1967) when they concluded that SPCAs 'do not have the characteristics of endarteries for they anastomose freely with each other and communicate as well with recurrent branches from the long posterior ciliaries, the anterior ciliaries, and vessels from the major circle of the iris'. This view has been shared by a majority of the workers in the field. In the literature interarterial and arterio-venous anastomoses in the human choroid have been described after post-mortem injection studies; however, it has been suggested by others that the choroidal arterial supply is segmental in vivo (Hayreh and Baines, 1972a).

In my in vivo experimental studies in rhesus monkeys, involving cutting of the PCAs (Hayreh, r973b; Hayreh and Baines, 1972a), SPCAs (Hayreh, 1974c) and long PCAs (Hayreh, 1974d) and investigating the choroidal circulation with fluorescein angiography, I found that these arteries do not anastomose at any level with any neighbouring artery, and are functional end-arteries. My studies (Hayreh, 1974b) also demonstrated that lobules of choriocapillaris supplied by each terminal choroidal arteriole show no functional anastomosis in vivo with one another. These haemodynamic studies therefore place a contrary interpretation upon the statement of Ring and Fujino (1967) and the prevalent concept based on post-mortem studies, and demonstrate the in vivo segmental distribution in the choroid not only of the main PCAs but also of all their subdivisions right down to the terminal choroidal arterioles and the choriocapillaris. The border between any two neighbouring vessels, from the main PCAs down to the terminal choroidal arterioles, forms a watershed zone. The watershed zone between the main PCAs usually passes through the optic disc, as shown in Figs 2 to 7. The watershed zones between the various temporal SPCAs and also those of the temporal long PCA are shown 
schematically in Fig. 7. The watershed zones between the various units of the choriocapillaris are shown in Fig. 9. The post-mortem studies,

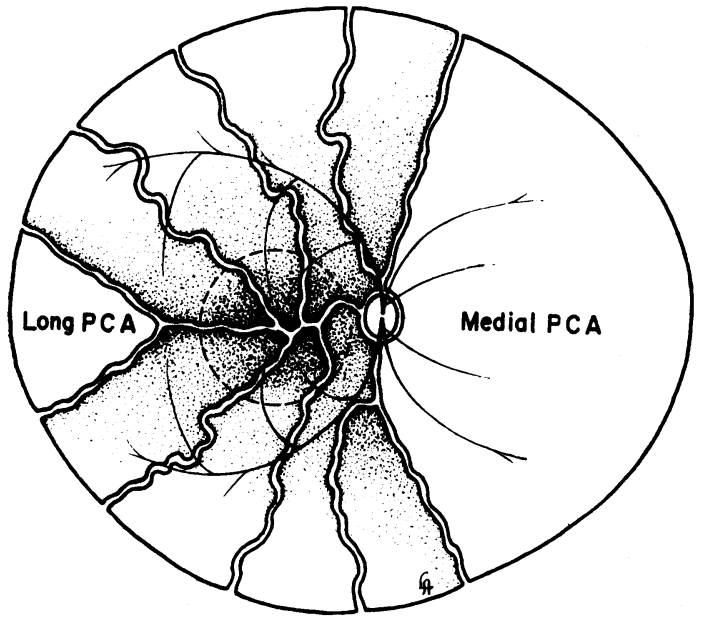

FIG. 7 Diagrammatic representation of distribution by various temporal SPCAs and their watershed zones in posterior part of fundus. Dotted circle in region of distribution of temporal SPCAs represents macular region. Areas of supply by medial PCA and temporal long PCA are also shown although extremely useful, have misled us to some extent about the actual haemodynamics in the choroid in general, and of the choriocapillaris in particular.

The peripapillary choroid, via recurrent pial branches in the pia of the retro-ocular optic nerve was found to establish anastomoses with pial branches from other sources (Hayreh, 1963b). In experimental occlusion studies of the PCAs (Hayreh, 1973b; Hayreh and Baines, 1972a), we found a filling of the peripapillary choroid via these anastomoses. This was also seen in similar studies by Anderson and Davis (1974). However, no such peripapillary choroid filling was ever seen in patients with PCA occlusion and consequent anterior ischaemic optic neuropathy (Hayreh, 1974g, 1975). This discrepancy between the clinical and experimental findings is discussed below (p. 64I).

\section{POSTERIOR CILIARY ARTERY DISTRIBUTION}

These arteries supply the following tissues:

\section{The choroid}

SPCAs supply the choroid presumably as far forward as the equator of the eyeball. Because of

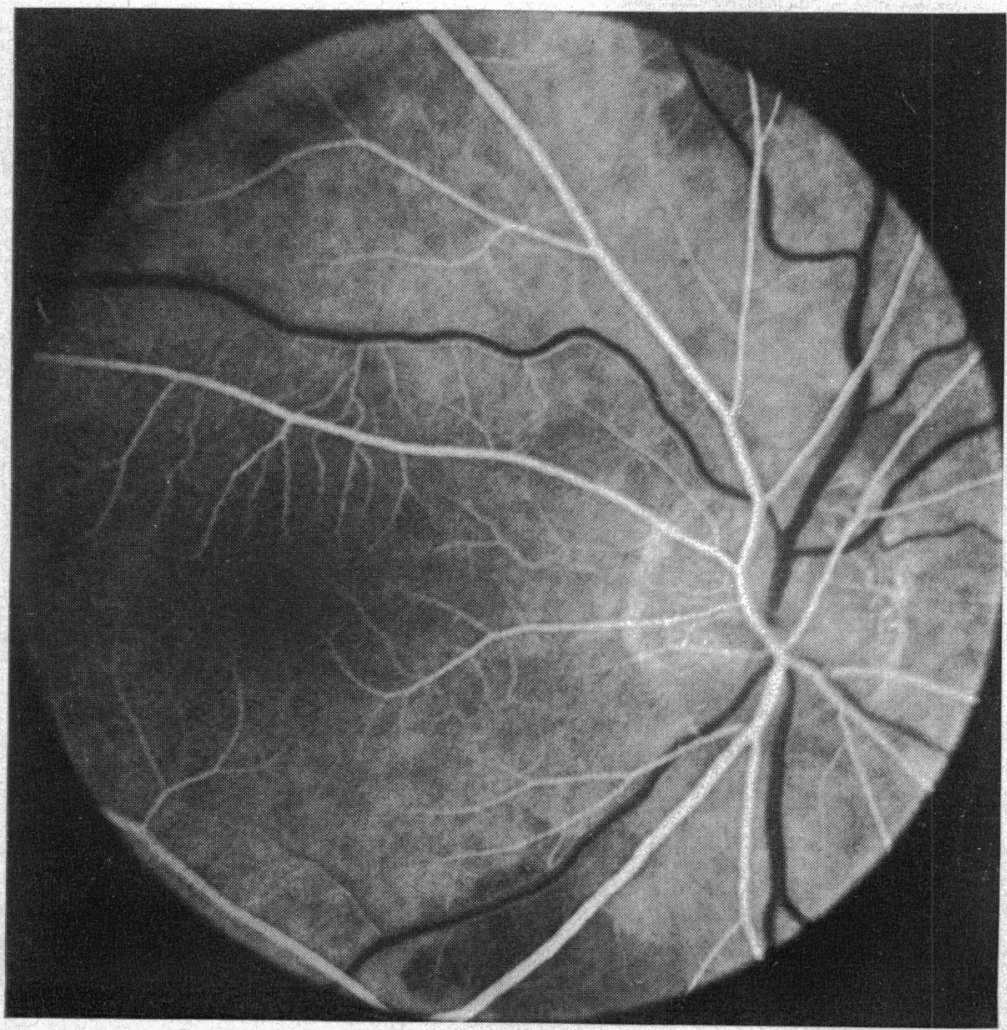

FIG. 8 Fluorescein fundus angiogram of normal human eye, showing three geographical filling defects in choriocapillaris bed 


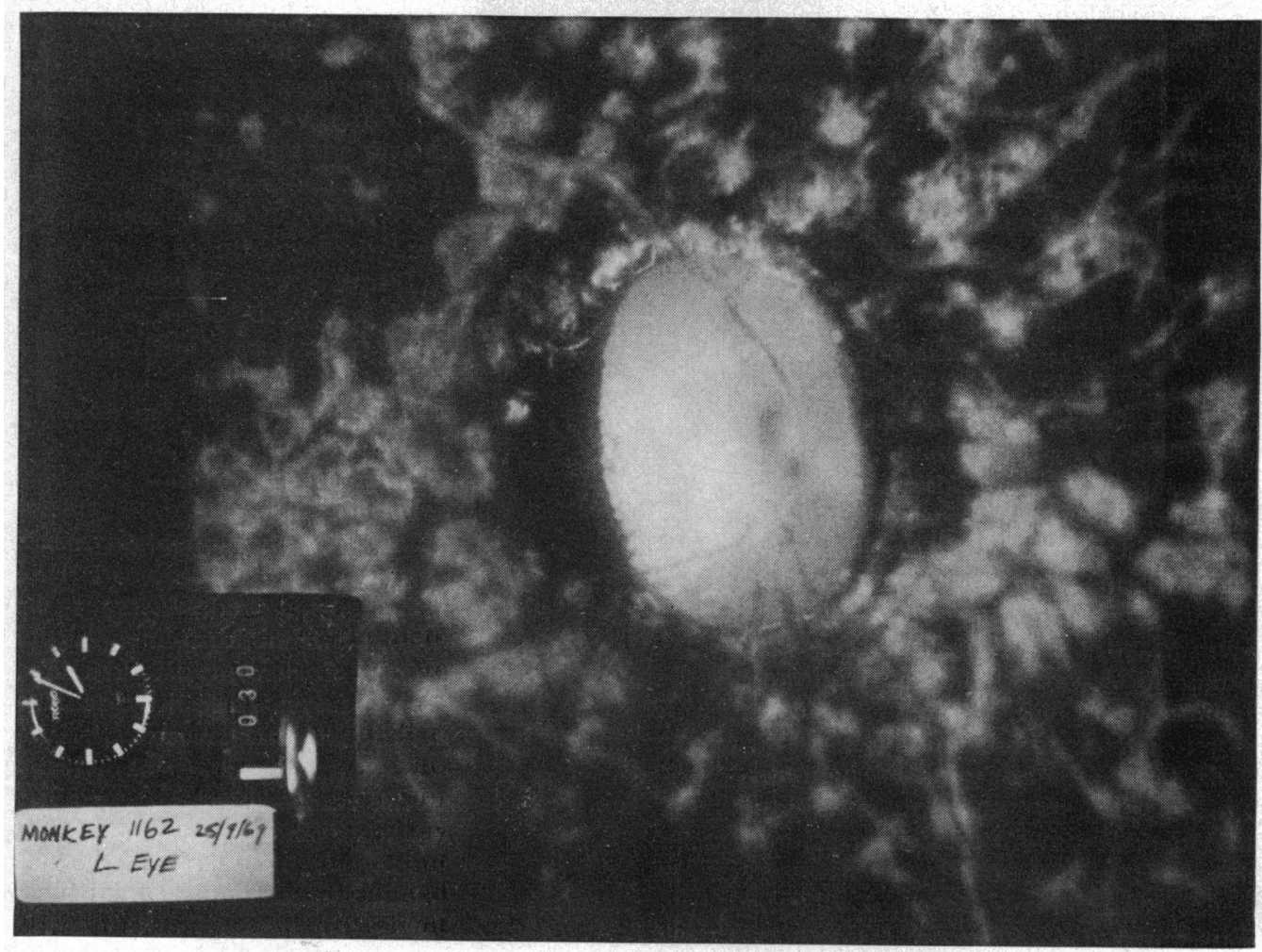

Fig. $9 b$

(a) Showing various units of choriocapillaris mosaic (each unit supplied by terminal choroidal arteriole) : note presence of some empty units among normally filled units (compare with filling defects in Fig. 8)

(b) and (c) Two consecutive angiograms of monkey eye showing : (b) Earliest arterial filling of choriocapillaris and their feeding terminal choroidal arterioles; (c) Venous phases of choriocapillaris filling with honeycomb pattern. Fluorescence of optic disc is due to earlier injection of fluorescein

Note that the time interval between (b) and (c) is about $0.8 \mathrm{~s}$, indicating tremendous speed of normal circulation in choriocapillaris and choroid. This is because wide lumen of choriocapillaris offers much less resistance to blood flow than retinal or any other capillaries

FIG. $9 a$

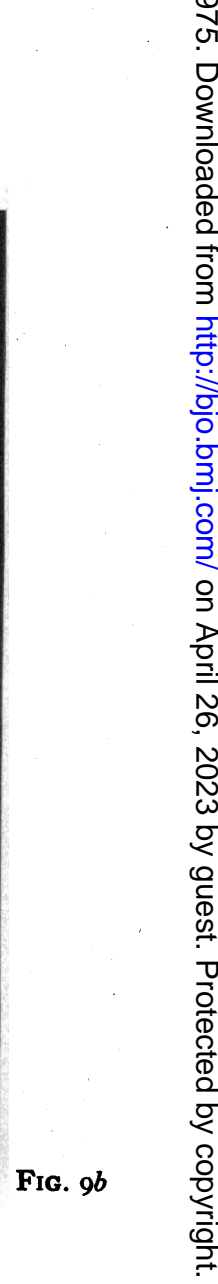




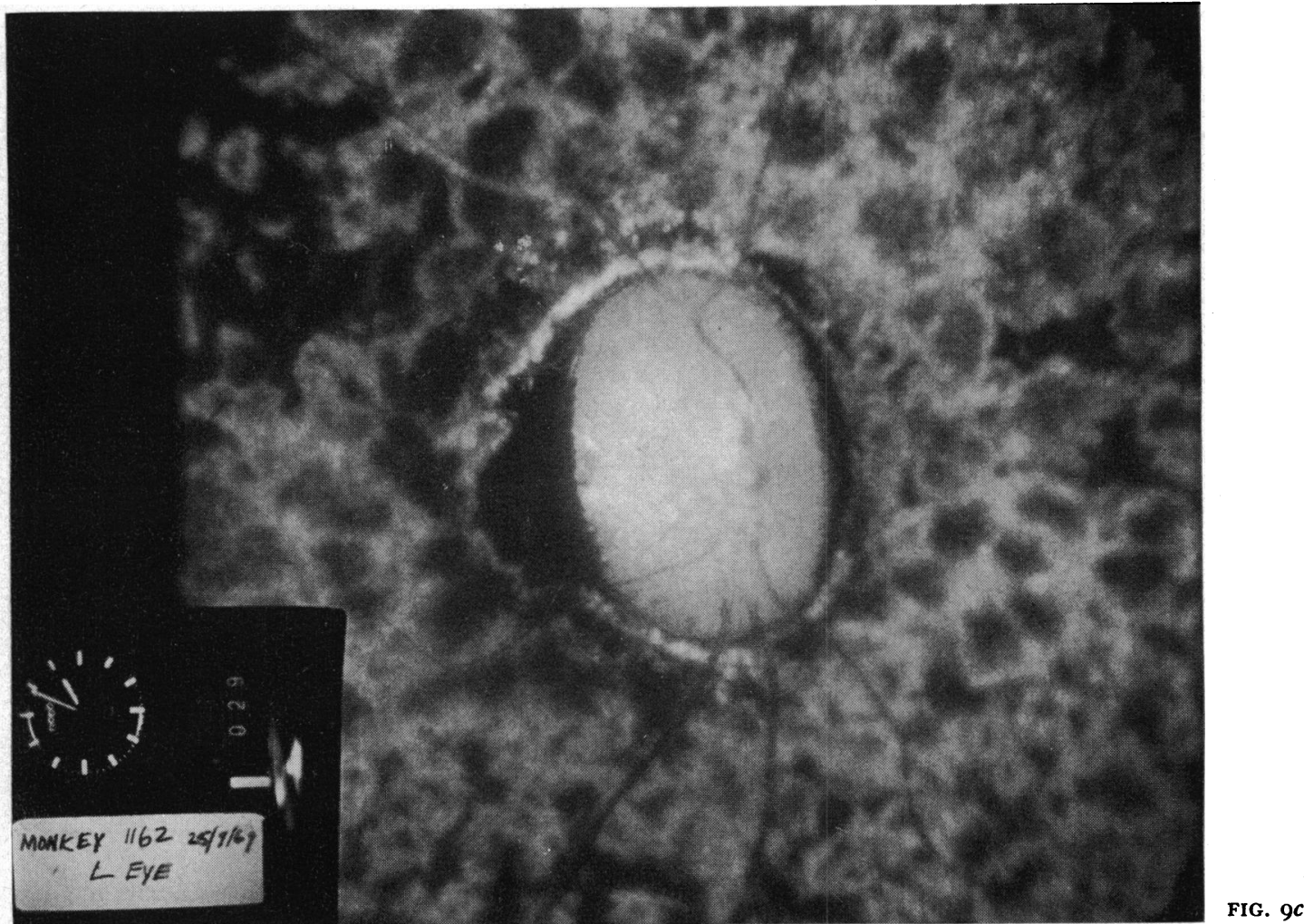

limitations of the ordinary fundus camera in photographing the fundus near the equator and anterior to that, it has not been possible to investigate the supply by the SPCAs in the equatorial region and in front of it. In studies involving occlusion of the PCAs (Hayreh, 1973b; Hayreh and Baines, 1972a) and SPCAs (Hayreh, 1974c), no retrograde filling of the choroid from the equator backwards was ever recorded, demonstrating the absence of any functional anastomoses between the SPCAs and the anterior choroidal arteries. The long PCAs, in addition to their contribution to the posterior choroid (Hayreh, 1974d) (Fig. 7), also supply the choroid anterior to the equator because of their final destination in the ciliary body.

\section{The retina}

The choroid supplies the overlying retina to a depth of about $130 \mu \mathrm{m}$ which includes the pigment epithelium layer, the layer of rods and cones, the outer nuclear and plexiform layers, and the whole thickness of the foveal retina. In eyes with cilioretinal arteries (Fig. 2), a variable area of the inner layers of the retina is also supplied; a cilio-retinal artery may supply an area of the retina varying from a tiny insignificant piece to as much as half and, rarely, even the entire retina (Hayreh, r963a). In some animals, such as cats, the entire blood supply to the retina is via the cilio-retinal arteries because of their lack of a central retinal artery. Thus the PCAs have a very important role to play in the blood supply of the retina.

Anterior part of the optic nerve (including the optic nerve head)

The PCAs are the only source of blood supply to the lamina cribrosa and prelaminar regions, and the main (if not the only) source to the retrolaminar region, and may supply the surface nerve fibre layer of the optic disc in some instances. The prelaminar region is mainly supplied from the peripapillary choroid (Figs 3 to 6). The subject of blood supply of the anterior part of the optic nerve is fully discussed elsewhere (Hayreh, 1969, 1974a, 1975).

\section{Anterior uveal tract}

The long PCAs play a role in the blood supply of the anterior uvea-that is, iris, ciliary body, and anterior part of the choroid.

The blood supply in the choroid and anterior 


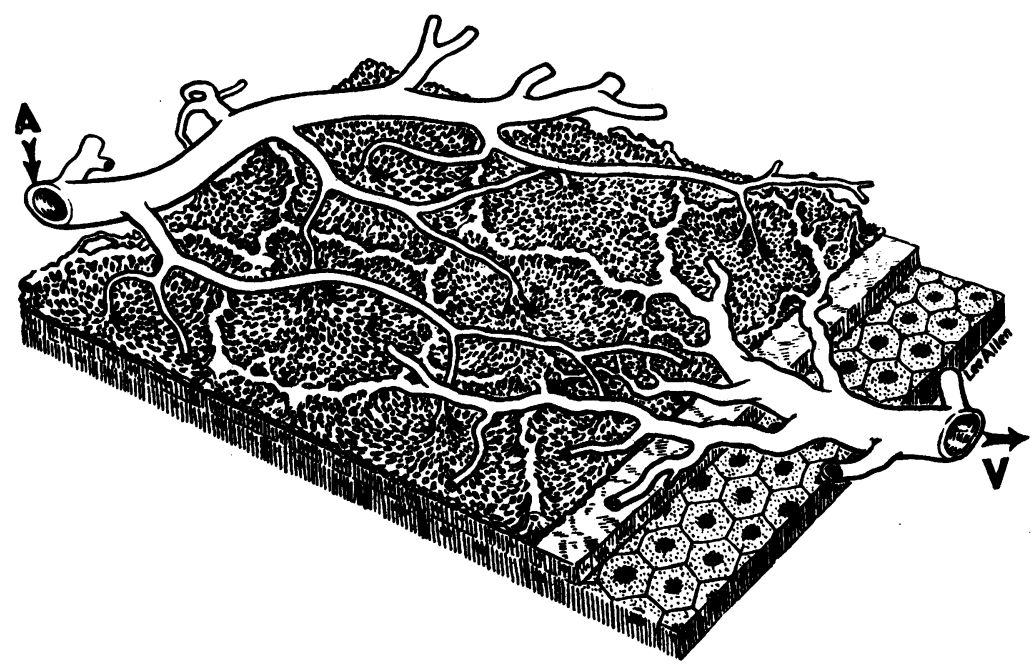

FIG. Io Three-dimensional schematic representation of choriocapillaris flow pattern. $A$ choroidal arteriole; $V$ choroidal vein

part of the optic nerve by the PCAs and their subdivisions, down to the terminal arterioles, is segmental. The blood supply by the long PCA is segmental in the choroid posterior to the equator, and presumably also in the anterior uvea.

\section{Venous drainage of the uveal vasculature}

It is generally assumed that the uveal veins freely communicate and that the vortex veins have no segmental distribution. Our in vivo studies in rhesus monkeys (Hayreh and Baines, 1973) revealed that each vortex vein has a well-defined segmental distribution extending throughout the entire antero-posterior length of the uveal tract involving the veins in the corresponding segment of the iris, ciliary processes, and the choroid. Occlusion of one vortex vein, therefore, produced marked localized venous engorgement in the corresponding segment of the iris, ciliary processes, and the choroid without involving veins in the rest of the uveal tract. The watershed zones between the various vortex veins, therefore, extend anteroposteriorly through the entire length of the uveal tract, a horizontal watershed between the upper and lower vortex veins passes through the optic disc and macular region, while a vertical watershed between the temporal and nasal vortex veins passes in between the optic disc and macular region (Fig. II). This divides the entire uveal venous system into four functionally independent segments; if the number of vortex veins is less or more than the usual four, the number of segments would also change accordingly.

\section{Clinical significance of segmental distribution of uveal vasculature}

The various aspects of this subject are discussed in detail elsewhere (Hayreh, 1973b, 1974b-g,
1975; Hayreh and Baines, 1972b, c, 1973). Vascular occlusive disorders of the choroid are considered here under the three following headings:

a. Arterial occlusive disorders

b. Choriocapillaris occlusive disorders

c. Venous occlusive disorders.

OCCLUSIVE DISORDERS OF THE POSTERIOR CILIARY ARTERY AND ITS SUBDIVISIONS

It has been demonstrated above that the arterial supply to the choroid is segmental from the PCAs right down to the choriocapillaris. However, our experimental occlusion of the various PCAs in rhesus monkeys did not produce infarction of the entire choroid supplied by the occluded PCA: instead there were only small patchy choroidal infarcts distributed in the territory of the occluded artery, and sometimes, indeed, no lesion at all in the choroid (Hayreh, 1973b; Hayreh and Baines, 1972b). On occluding the SPCA and long PCA, no choroidal infarct was usually seen in experi-

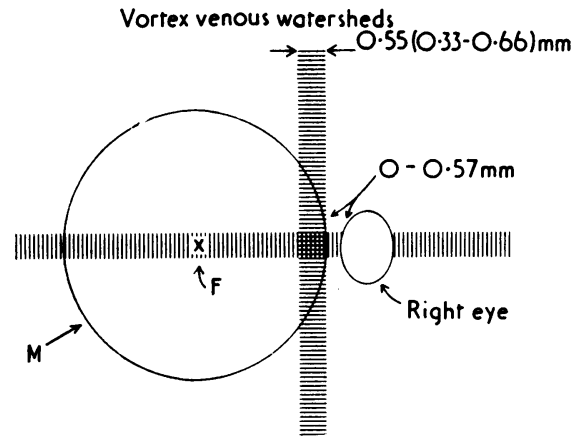

FIG. I I Diagrammatic representation of watershed zones of vortex veins. $F$ and $X$ fovea; $M$ macular region; $O D$ optic disc 
ments with rhesus monkeys. These findings seem to conflict with the above-mentioned concept of segmental supply by the choroidal arteries. It is, therefore, imperative to discuss the various factors involved in these discrepancies.

I. There is a very important difference between these experimental occlusions in rhesus monkeys and those occurring as a disease process in humans. All the animals used in experimental studies were young and healthy; their age corresponded to that of a 10 to 15 -year-old human or younger. The normal healthy vascular bed, in almost all of the body of young, healthy individuals, has a great potential to develop quick collaterals and it is extremely difficult to produce any massive infarctions. By contrast, in elderly arteriosclerotic individuals with diseased vasculature, the response to similar arterial occlusion would be totally different. In an attempt to produce experimental ocular ischaemia (Henkind, 1974), similar to that seen in pulseless disease, a common carotid artery was ligated in a dog; this failed to produce any ischaemia. After some time the second common carotid artery was ligated, also with no effect. Then one vertebral artery, followed later by the second, was ligated but the animal still did not develop ocular or cerebral ischaemia, because of quickly developed collaterals. In elderly individuals, on the other hand, it is well known that ligation of one or two of these arteries would be catastrophic. Similarly, in my young monkeys, I have on many occasions ligated the femoral and axillary arteries high up while cannulating them without producing any ischaemia in the limbs, although similar arterial occlusions in elderly humans would produce massive gangrene. From these and many similar reports in the literature, and from the development of prominent extraocular anastomoses after PCA occlusion in rhesus monkeys (Hayreh and Baines, 1972a), it seems logical to conclude that occlusion of the various types of PCAs, which produces minor choroidal lesions or none in young, healthy monkeys, would produce a marked ischaemic lesion in an old, arteriosclerotic human patient. This is an important consideration to be borne in mind.

2. Perfusion of intraocular vessels is dependent upon the perfusion pressure (perfusion pressure $=$ mean blood pressure minus intraocular pressure). In experimental studies involving occlusion of the PCA, SPCA, and long PCA by orbitotomy there was a marked fall in intraocular pressure, which became unrecordably low 1 or 2 days after the occlusion (Hayreh, 1974d, Hayreh and Baines, 1972b). Such a marked fall in the intraocular pressure would assist the filling of the occluded segment of the choroid from the channels mentioned below. The perfusion of the occluded choroid in eyes with normal intraocular pressure would be much less than that in eyes with unrecordably low pressure. This is another important difference between these experimental occlusions and those occurring in patients.

3. The segment of the choroid supplied by an artery does not correspond exactly with the segment drained by a large choroidal vein; there is always a certain amount of overlap between the adjacent arterial segments via the veins. For example, the mechanism of retrograde flow into the occluded choroid, when one of the SPCAs is occluded, is shown diagrammatically in Fig. 12. This shows two adjacent segments of the choroid supplied by two arteries-the artery to the upper segment is patent while that to the lower segment is occluded. The blood from the patent artery fills the choriocapillaris in its territory and drains into the corresponding vein which joins the vein from the occluded segment. The common vein thus drains one normally filling segment and one occluded segment of the choroid. Since the blood pressure in the vein from the occluded segment is practically zero, some blood from the large vein regurgitates into the empty vein and partially fills the empty segment. This was seen on fluorescein angiography in all experimental occlusions of the SPCAs. Most probably this reflux of blood from a large vein into the occluded segment is due to the pumping action produced by ocular pulsation.

Fig. 13 illustrates diagrammatically what happens when an MPCA or LPCA is occluded. The territory of the uveal tract drained by one vortex vein is usually supplied by two PCAs

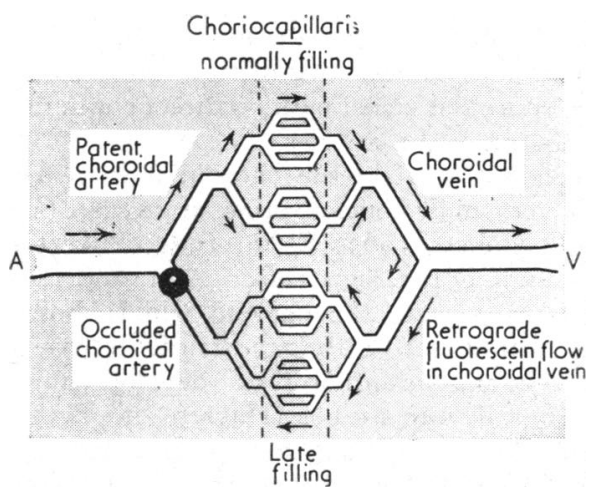

FIG. I2 Diagrammatic representation of mechanism of retrograde filling of choroid in area of an occluded short $P C A$ 
(to the posterior uvea) and a large number of of the anterior ciliary arteries (to the anterior uvea). Thus, occlusion of the MPCA or LPCA reduces the blood pressure in the venous tributaries draining the non-filling sector of the choroid. Blood would regurgitate from the main stem of the vortex vein into these tributaries and lead to retrograde filling of the choroid. This is supported by the fact that in the occluded territory isolated areas of the choroid start to fill with fluorescein on angiography during the late venous phase. These fluorescent patches are not continuous with any part of the normally filled choroid (Hayreh and Baines, 1972a). The normal ocular pulsation, by acting as a pumping mechanism, would help in filling and emptying the veins from the occluded choroid. When the main PCA is occluded, the occluded area of the choroid is so large that there is not enough overlap between the occluded and unoccluded segments by the mechanism mentioned above, and hence fundus lesions are almost always seen. Thus the various arterial segments communicate via the choroidal veins, with no direct communication between the arteries.

4. The venous blood in the choroid is known to have a very high concentration of oxygen (Cohan and Cohan, 1963; Elgin, 1964; Pilkerton, Bulle, and O'Rourke, 1964; Alm and Bill, 1970). Any part of the choroid which showed any perfusion during the transit of the dye on fluorescein angiography did not develop choroidal lesions in our studies (Hayreh and Baines, r 972b); thus indicating that a slow trickle of venous choroidal blood was enough to supply the required amount of oxygen. It was interesting to observe that in our experimental PCA occlusion, elongated pointed lesions occurred with narrow intervening strips of normallooking fundus (Hayreh, 1973b; Hayreh and Baines, 1972b) (Fig. 14); the latter were mostly overlying large choroidal veins. This would indicate that diffusion of oxygen through the thin-walled choroidal veins was enough to prevent ischaemic damage in the overlying tissue.

5. Other extraocular anastomoses which help to fill the occluded choroid are described elsewhere (Hayreh and Baines, 1972a). The blood which comes through these anastomoses also reaches the choroid by the venous phase of the transit of dye, and is thus of a venous nature and has sufficient oxygen in it.

The interpretation of the effects of occlusion of the PCA or its further subdivision must therefore be considered in the light of these important factors.

The following clinical lesions are seen in eyes with occlusion of the PCA which is usually due to arteriosclerosis but may occur in other vascular diseases-such as hypertension, collagen disease, temporal arteritis, diabetes, syphilis, hypercholesterolaemia, thromboangiitis obliterans, Raynaud's disease, migraine, pulseless disease, embolism, and various haematological disorders.

\section{(a) Chorio-retinal lesions}

In our studies of PCA occlusion, we described the development of patchy chorio-retinal lesions in the fundus situated posterior to the equator and in the

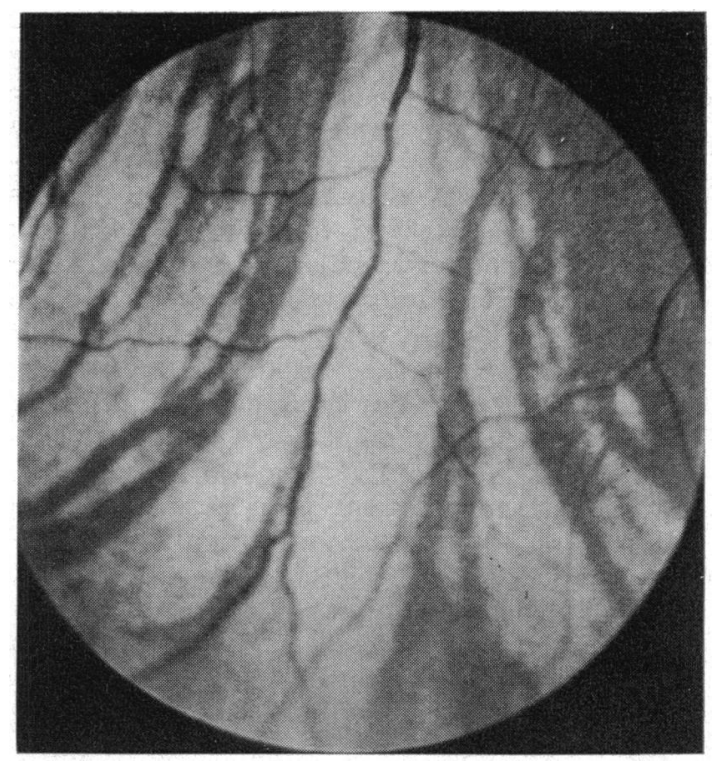

FIG. I4 Fundus photograph of monkey, showing white chorio-retinal lesions 2 days after occlusion of LPCA. Note absence of lesion over major choroidal veins 
part supplied by the artery (Hayreh, 1973b; Hayreh and Baines, 1972b). These were due to infarction of the pigment epithelium and the outer retina (Fig. 14). Within 24 hours after the occlusion, white patches of markedly varied shape, size, and distribution were seen; after 2 to 3 weeks these became greyish-white, granular, and depigmented areas. Similar chorio-retinal lesions have been described in patients (Hepburn, 1935; Amalric, 1971 ; Foulds, Lee, and Taylor, 1971). Presumably many of the segmental chorio-retinal degenerative lesions of obscure aetiology and some of the atypical sectoral or uniocular retinitis pigmentosas belong to this group.

\section{(b) Anterior ischaemic optic neuropathy (AION)}

I have investigated the subject recently and discussed it at length elsewhere (Hayreh, I974f, g, 1975). In AION there is a comparatively sudden loss of vision in the entire eye or in one sector of the field of vision of the eye, initially associated with oedema of the optic disc, which resolves to optic atrophy within a month or two, leaving a permanent visual defect. Its pathogenesis has been the centre of a good deal of controversy. My clinical (Hayreh, 1974f, g, 1975) and experimental (Hayreh and Baines, I972C) studies, and a review of histopathological findings in such eyes reported in the literature (Henkind, Charles, and Pearson, 1970) show that AION is due to occlusion of the PCAs. Recently, Anderson and Davis (1974), from experimental occlusion of the PCAs in II Squirrel monkeys, concluded that the AION 'must represent disease of more than just the PCAs' because only minute areas of atrophy in the optic nerve head were found instead of the large areas of infarction seen in patients. I have discussed the fallacy of such a statement elsewhere (Hayreh, 1975) and it can be understood if one considers the following differences between experimental studies and patients with AION.

I. The age of the monkey used in the experimental PCA occlusion by us (Hayreh and Baines, 1972c) and by Anderson and Davis (1974) is comparable with an age of 10 to 15 years or younger in humans. AION, however, is a disease of persons who are past middle age with arteriosclerosis, arterial disease, hypertension, etc. The importance of this age factor is discussed on p. 64r.

2. The other important complicating factor in experimental PCA occlusion as compared with the PCA occlusion occurring in patients is the marked fall in intraocular pressure to unrecordably low levels in the monkeys after lateral orbitotomy (Hayreh, 1974d; Hayreh and Baines, 1972b) (see p. 64r).
We have demonstrated clearly that PCA occlusion in young monkeys immediately opens up the anastomoses between the peripapillary choroid and the pial plexus on the optic nerve via the recurrent pial branches from the peripapillary choroid (Hayreh and Baines, 1972a). The perfusion of vessels in the peripapillary choroid and optic nerve head further improves markedly after the above-mentioned fall in intraocular pressure (Hayreh, 1975). This explains the frequent absence of marked damage to the anterior optic nerve in animals. On the other hand, a similar or even much less severe occlusion of the PCAs in elderly arteriosclerotic persons with normal intraocular pressure and diseased arteries would produce massive infarction (Fig. 3). Evidently Anderson and Davis (1974) did not take these additional factors into consideration in reaching their conclusions.

\section{Role of the choroidal blood supply in macular lesions}

The localized involvement of the macular region by a large number of conditions is well known and this has naturally excited a good deal of curiosity about the reasons for this marked vulnerability of the macular region to degenerative disease. I have discussed the submacular choroidal vascular pattern and its possible role elsewhere (Hayreh, I974c). It may be stated at the outset that we have so far no definite evidence of the existence of a special artery to the submacular choroid, despite some claims (p. 634). My in vivo studies in monkeys showed that the macular region is the meeting point not only of many watershed zones of the various SPCAs (Fig. 7), but also of all the watershed zones of the vortex veins (Hayreh and Baines, 1973) (Fig. II).

To understand the clinical significance of the fact that the macular region of the retina is located at the meeting-place of the watershed zones of the SPCAs, one must discuss the circulatory disorders of the watershed or boundary zones. Of all the various endarterial systems with watershed zones in the body the cerebral circulation has been investigated most thoroughly. It has been known for some time that the watershed zones between large arterial systems are more vulnerable to ischaemia or anoxia than others in the brain (Blackwood, McMenemey, Meyer, Norman, and Russell, 1963). This phenomenon was first demonstrated by Lindenberg and Spatz (1940) and has subsequently been described by many workers (Meyer, 1953; Zülch, 1953; Norman, Urich, and McMenemey, 1975; Zülch and Behrend, 196r). The haemodynamic peculiarities of these watershed zones were discussed by Eich and Wiemers (1950). Schneider (1956), while discussing the special vulnerability of the watershed zones of the vascular tree to 
ischaemia, compared the process to failure of a farm irrigation system in which 'the meadows at the end of the line suffer most when the main pipe supplies too little water'. When there is a generalized reduction of blood supply to the brain, ischaemia is felt most in the most distal part of the irrigation areas-that is the watershed zones (Alajouanine, Castaigne, Lhermitte, Cambier, and Gautier, 1959). This process, in the German literature, has aptly been called wipfeldüre, which literally means withering away of the peripheral twigs of a tree because nutrition is not reaching them (Fig. 15). In the cerebral circulation it is known that ischaemic anoxia of the terminal vascular net results in small vessel changes, particularly at the junction of the watershed zones of the principal cerebral arteries, and it has been stated that cerebral ischaemia may occur in spite of a continuing circulation, because it is insufficient to meet the metabolic needs of the tissue (Meyer, 196I). It has also been shown experimentally in monkeys that anastomotic cerebral circulation does not become established if the blood pressure in the arterial field surrounding the ischaemic zone falls below $60 \mathrm{~mm} \mathrm{Hg}$ (Meyer, Fang, and Denny-Brown, 1954). In the event of a fall of blood pressure in the cerebral circulation, it can be presumed that the blood supply may be adequate for the proximal but not for the distal territories; this, however, is not so, because the proximal supply may be more severely affected than the distal (Norman and others, (957).

From the observations of the wateshed zones of the cerebral circulation, it would seem logical to consider that the macular region in the eye, where numerous watershed zones of the SPCAs (Fig. 7) and of the vortex veins (Fig. II) meet, would be highly vulnerable to ischaemic disorders in any generalized chronic ischaemic disorder of the choroid. This process may be compared with that illustrated in Fig. 16, where, on a fall of water pressure in the garden sprinklers, the central area which previously received an adequate water supply from all the surrounding sprinklers, has dried up. A reduction and even disappearance of the choriocapillaris in the macular region which may be seen in senile macular degeneration is shown schematically in Fig. 17. It is postulated (Hayreh, 1974c) that senile macular degeneration, senile disciform macular degeneration, and allied macular disorders are most probably due to this unusual pattern of the submacular choroid, compared with the rest of the choroid. Similarly, pigmentary and other degenerations seen in old persons in the peripheral part of the fundus may represent an ischaemic phenomenon in the watershed zones between the short posterior and the anterior ciliary arteries.
Role of the long PCA in anterior segment ischaemia

The occurrence of anterior segment ischaemia after diathermy, scleral buckling, and some other procedures for retinal detachment has been attributed to interference with the long PCAs, so that

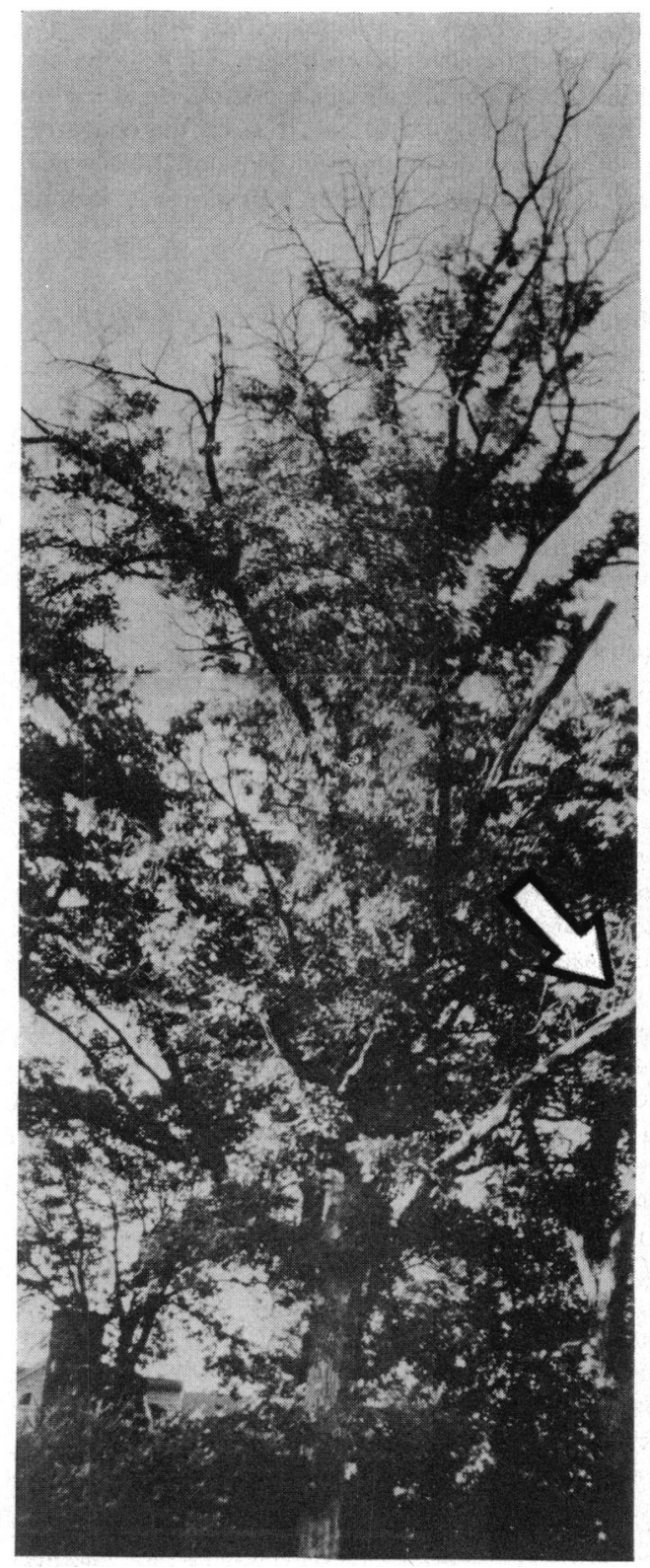

FIG. I Photograph of tree showing phenomenon of wipfeldüre (withering away of peripheral twigs because nutrition is not reaching them). Note that even one of the lower branches has completely withered (arrow); this is comparable with early macular branches from SPCAs 


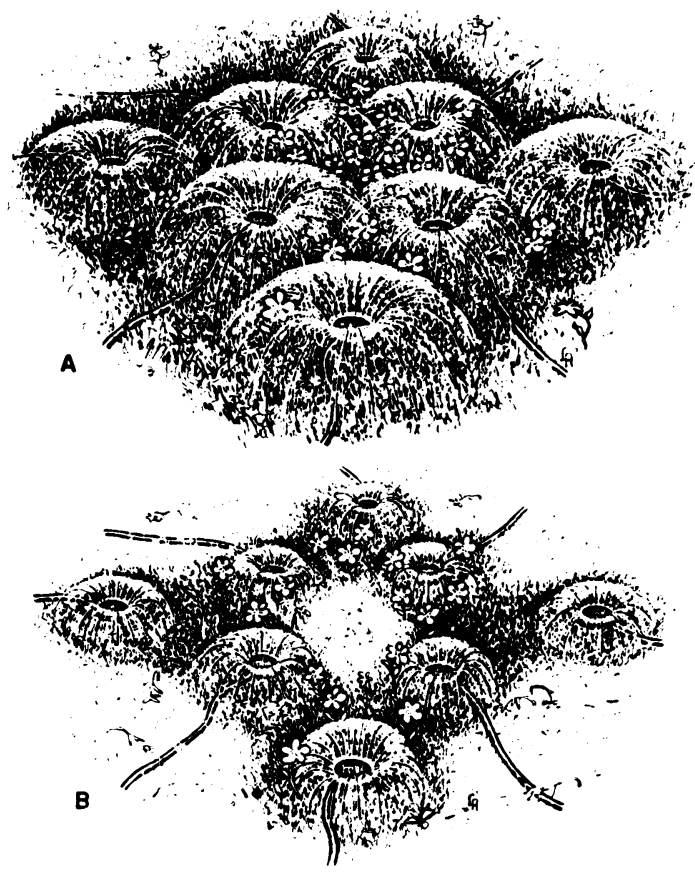

FIG. I6 Two illustrations demonstrating effect of reducing water pressure in eight garden sprinklers watering a lawn

(a) Watering at normal water pressure

(b) Same area supplied by sprinklers with reduced water pressure. Note that in area where water supply by various sprinklers meets in centre, lawn has dried up, as have peripheral parts of lawn although intermediate zone is still normal

these arteries have become a 'sacred cow' for ophthalmologists. My studies of the in vivo effects of occlusion of the long PCAs in rhesus monkeys (Hayreh, 1974d) revealed that occlusion of the long PCAs produces no significant effect on the anterior segment. The sacred-cow status of the long PCAs is based on previous studies in rabbits in which the long PCAs are the only source of blood supply to the anterior segment-whereas in primates and man the anterior segment is supplied by the anterior ciliary arteries (usually seven in number) and the long PCAs (two in number).

Many unsuccessful attempts have been made in human subjects to find out if occlusion of the long PCA would help to control the raised intraocular pressure in glaucoma; this subject is reviewed elsewhere (Hayreh, 1974d).

\section{OCCLUSIVE DISORDERS OF THE CHORIOCAPILLARIS}

It is known that choroidal lesions-particularly inflammatory, metastatic, and degenerative lesions -tend to be localized. The segmental nature of the choriocapillaris already described (p. 635) helps to explain such localization. Elschnig's spots,
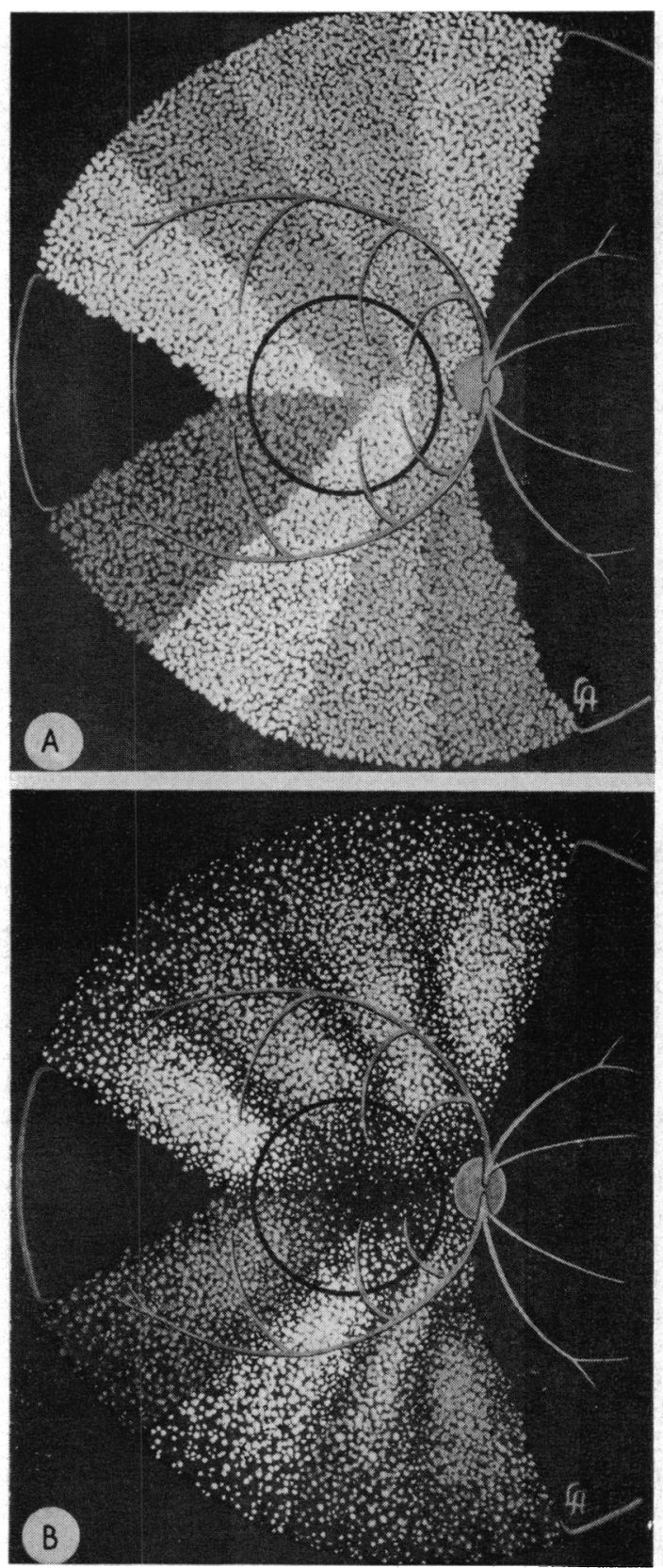

FIG. 7 Diagrammatic representation of distribution of choriocapillaris supplied by various temporal SPCAs (a) Normal pattern

(b) Postulated pattern caused by generalized chronic ischaemic disorder of choroid, with reduction of choriocapillaris most marked in macular region and equatorial choroid (compare Fig. 16b) 
Siegrist's streak, and other allied lesions in malignant hypertension and associated diseases, and in collagen disease (for example, polyarteritis nodosa), represent pigment epithelium degenerative changes over a segment of the choriocapillaris, which are due to fibrinoid necrosis and obliteration of the choroidal arteriole and its segment of the choriocapillaris. Microsphere embolization of choroidal arteries in dogs (Golder and Gay, 1967) and in cats (Henkind, 1967) produced similar fundus lesions. The shapes and sizes of many of the conditions included in the category of 'flecked retina syndrome' (Krill and Klien, 1965) very much resemble the choriocapillaris pattern. For example, lesions in so-called Doyne's honeycomb dystrophy correspond to the arterial part of the choriocapillaris units (Figs 9 and 18) and suggest that they may be due to a disorder of the arterial segment of the choriocapillaris. In fundus flavimaculatus the shape and distribution of the 'fish tail' lesions resemble venous segments of the choriocapillaris units and suggest that this may be a disorder of the venous part of the choriocapillaris unit. A vascular basis for the flecked retina syndrome is further suggested by the occurrence in the mid and far periphery of the fundus of chorio-retinal lesions (Newell, 1975) similar to those seen in choroidal ischaemia. However, the inheritance patterns seen in this syndrome are hard to explain on a vascular basis.

I feel that so-called 'acute posterior multifocal placoid pigment epitheliopathy' (Gass, 1968) represents an occlusive disorder of the terminal choroidal arteriole because each focus in this disease has a close resemblance in size and shape to a unit of choriocapillaris supplied by a terminal choroidal arteriole. The initial whitish focus probably represents pigment epithelial infarction and the later depigmented spots probably represent pigment epithelial degeneration overlying the occluded units of the choriocapillaris (similar to that seen in our experimental posterior ciliary artery occlusion-Hayreh, 1973b: Hayreh and Baines, 1972b). However, the cause of the occlusion still remains obscure. Similarly the fluoresceinleaking spot seen on fluorescein angiography in central serous retinopathy, and the multiple fluorescent spots in Harada's disease, seem to correspond to individual choriocapillaris units (Fig. 9).

\section{OCCLUSIVE DISORDERS OF THE VORTEX VEINS}

The clinical picture of retinal venous occlusion is well known, but no corresponding description of vortex vein occlusion in man has previously been available. Following on my experimental studies, involving in vivo occlusion of the vortex veins in rhesus monkeys (Hayreh and Baines, 1973), I have presented a clinical picture of such an occlusion, and discussed its possible clinical significance as well as the role of segmental distribution by the vortex vein in its production. The findings strongly suggest that interference with the vortex vein circulation (and its segmental distribution) could play an important role in the pathogenesis of some of the major complications of retinal detachment surgery-for example, glaucoma, anterior uveitis, ischaemia and necrosis of the anterior segment, and choroidal and intraocular haemorrhages. Experimental vortex vein occlusion produced in all eyes a thick flare or thick gelatinous deposit in the anterior chamber and hyperaemia of the iris (this mimics perfectly the non-granulomatous iritis of sudden onset), and in some eyes hyphaema.

\section{Summary}

The various in vivo studies on posterior ciliary arteries and choroidal vasculature reported piecemeal by the author over the past few years have now been collated. A coherent picture of the vasculature emerges which is of considerable clinical significance. The observations show that the posterior ciliary arteries and their branches right down to the terminal choroidal arterioles, the choriocapillaris, and the vortex veins have a segmental distribution 
in the choroid, and that the posterior ciliary arteries and choroidal arteries function as end-arteries. No special macular artery supplying the submacular choroid has been found. The posterior ciliary arteries not only supply the choroid but are also the main source of blood supply to the anterior part of the optic nerve, and have an important role in the blood supply of the retina. The clinical significance of this segmental distribution of the uveal vasculature is discussed.

It is a pleasure to acknowledge the help of my wife, Shelagh, in the preparation of the manuscript and of $\mathbf{M r}$ Lee Allen for drawing Figs 7, 10, and 12, and of Ms Vicky Dingman for secretarial assistance. Figures 7-I I are reproduced by courtesy of the editor of Albrecht v. Graefes Archiv für Ophthalmologie.

\section{References}

alajouanine, t., Castaigne, P., lhermitte, f., Cambier, J., and gautier, J. c. (1959) Sem. Hôp. Paris, 35, 1 i 49 ALM, A., and BILL, A. (1970) Acta physiol. scand., 80, 19

AMALRIC, P. (197I) Trans. ophthal. Soc. U.K., 91, 305

- (1973) Trans. Amer. Acad. Ophthal. Otolaryng., 77, OP-291

ANDERSON, D. R., and DAVIS, E. B. (1974) Arch. Ophthal. (Chicago), 92, 422

BLACKWOOD, W., MCMENEMEY, W. H., MEYER, A., NORMAN, R. M., and RUSSELl, D.s. (1963) In 'Greenfield's Neuropathology', p. 260. Williams \& Wilkins, Baltimore

cohan, B. E., and cohan, s. B. (1963) Amer. F. Physiol., 205, 60

DOLleRY, C. T., HENKIND, P., KOHNER, E. M., and PATERSON, J. W. (1968) Invest. Ophthal., 7, 191

DUKE-ELDER, s. (I961) In 'System of Ophthalmology', vol. 2, pp. 339, 346, 35 1. Kimpton, London

EICH, J., and WIEMERS, K. (1950) Dtsch. Z. Nervenheilk., 164, 537

ELGIN, S. S. (1964) Invest Ophthal., 3, 417

FOULDS, W. S., LEE, W. R., and TAYLOR, w. O. G. (I97I) Trans. ophthal. Soc. U.K., 91, 323

GASS, J. D. M. (1968) Arch. Ophthal. (Chicago), 80, 177

GOLDER, H., and GAY, A. J. (1967) Invest. Ophthal., 6, 5 I

HAYREH, s. S. (1962) Brit. F. Ophthal., 46, 212

(1963a) Ibid., 47, 7r

(1963b) Ibid., 47, $65 \mathrm{I}$

(1964) Exp. Eye Res., 3, 16

(1969) Brit. F. Ophthal., 53, 721

(1970) Ibid., 54, 289

- (1973a) Exp. Eye Res., 17, 387

(1973b) Trans. Amer. Acad. Ophthal. Otolaryng., 77, OP-300

- (1974a) Ibid., 78, OP-240

(1974b) v. Graefes Arch. Ophthal., 192, 165

(1974c) Ibid., 192, 181

- (1974d) Ibid., 192, 197

(1974e) Brit. F. Ophthal., 58, 391

(1974f) Ibid., 58, 955

(1974g) Ibid., 58, 964

(1975) In 'Anterior Ischemic Optic Neuropathy'. Springer-Verlag, New York

and BAINES, J. A. B. (1972a) Brit. F. Ophthal., 56, 7 I9 (1972b) Ibid., 56, 736

(1972c) Ibid., 56, 754

- (1973) Ibid., 57, 217

HENKIND, P. (1967) Invest. Ophthal., 6, 56

(1974) Personal communication

Charles, N. C., and Pearson, J. (1970) Amer. Y. Ophthal., 69, 78

HEPBURN, M. L. (1912) Trans. ophthal. Soc. U.K., 32, 36 r

(1935) Ibid., 55, 434

hogan, M. J., and Alvarado, J. A., and Weddell, J. E. (I97 I) In 'Histology of the Human Eye', p. 372.

Saunders, Philadelphia

KREY, H. F. (1975) Amer. F. Ophthal., 80, 198

KRILL, A. E., and KLIEN, B. A. (1965) Arch. Ophthal. (Chicago), 74, 496

LEBER, T. (1903) Die Circulations- und Ernährungsverhältnisse des Auges, in 'Graefe-Saemisch: Handbuch der

gesamten Augenheilkunde', Bd. 2, Abt. 2, pp. 33-35, 43-46. Engelmann, Leipzig

LINDENBERG, R., and SPATZ, H. (1940) Virchows Arch. path. Anat., 305, 531

MEYER, F. (1887) Morph. $¥ b$., I2, 414

MEYkR, J. E. (1953) Arch. Psychiat. Nervenkr., I90, 328

MEYRR, J. S. (1961) In 'Pathogenesis and Treatment of Cerebrovascular Disease', ed. W. S. Fields, p. 8o.

Thomas, Springfield, Ill.

-, FANG, H. C., and DENNy-BRown, D. (1954) Arch. Neurol. Psychiat. (Chic.), 72, 296 
NEWBLL, F. w. (1975) 'Variants of flecked retina syndrome', presented at roth Congreso Pan Americano de Oftalmologian, San Juan, Puerto Rico

NORMAN, R. M., URICH, H., and MCMENEMEY, W. H. (1957) Brain, 80, 49

PILKERTON, R., BULLE, P. H., and O'ROURKE, J. (1964) Invest. Ophthal., 3, 237

RING, H. G., and FUJINO, T. (1967) Arch. Ophthal. (Chicago), 78, 43I

ROHEN, J. W. (1965) Int. Ophthal. Clin., 5, 581

SCHNEIDER, M. (1956) Therapiewoche, 6, 217

TORCZYNSKI, E., and Tso, M. O. (1974) Abstracts, Ass. Res. Vision Ophthal., Spring Meeting Sarasota, Florida WEITER, J. J., and ERNEST, J. T. (1974) Amer. Y. Ophthal., 78, 583

wolfF, E. (1948) In 'The Anatomy of the Eye and Orbit', p. 68-72. Lewis, London

WYBAR, K. c. (1954a) F. Anat. (Lond.), 88, 94

(1954b) Brit. F. Ophthal., 38, $5^{13}$

zÜLCH, K. J. (1953) Zbl. allg. Path. path. Anat., 90, 402

and BEHREND, R.C.H. (1961) In 'Cerebral Anoxia and the Electroencephalogram', ed. H. Gastaut and

J. S. Meyer, p. 144. Thomas, Springfield, Ill. 